\title{
Behavioural treatment of slow cortical potentials in intractable epilepsy: neuropsychological predictors of outcome
} Irene Daum, Brigitte Rockstroh, Niels Birbaumer, Thomas Elbert, Anthony Canavan,
Werner Lutzenberger

\begin{abstract}
The study aimed to explore the predictive value of neuropsychological tests within the context of acquisition of slow cortical potential (SCP) self-control, a technique which has beneficial effects on seizure frequency in epilepsy. Patients with epilepsy who successfully achieved SCP control had longer digit or block-tapping spans than less successful patients. Patients who showed a better learning rate across training also displayed better verbal memory and learning abilities. Seizure reduction was related to block-tapping spans only. The results indicate that measures of attention, as indicated by digit spans or block-tapping spans, offer some predictive value for acquisition of SCP control and treatment outcome, whilst measures of visuospatial or frontal lobe function are unrelated to SCP acquisition and seizure reduction.
\end{abstract}

(F Neurol Neurosurg Psychiatry 1993;56:94-97)

Behavioural interventions have been shown to be successful in supplementing traditional drug therapy in epilepsy. ${ }^{1}$ Behavioural techniques have included self-perception and relaxation, ${ }^{2}$ conditioning of seizure behaviour ${ }^{3}$ and self-regulation of end-tidal carbon dioxide $^{4}$ or EEG frequency bands. ${ }^{5}$ A more recent approach involves instrumental conditioning of slow cortical potentials within a self-regulation paradigm. ${ }^{6}$ Slow cortical potentials (SCPs) may reflect cortical excitability. ${ }^{6}$ There is increasing evidence that extreme cortical negativities indicate overexcitability of cortical neuronal networks and a high risk for the development of epileptic seizures. ${ }^{67}$ Arguably, regulation and control over surface-recorded SCPs represents regulation and control over cortical excitability. ${ }^{7}$ A therapeutic approach aimed at reducing cortical negativity and stabilisation of SCPs may therefore prove to have a beneficial effect on seizure frequency. In the SCP self-control procedure, subjects receive on-line visual feedback of their surface-recorded SCPs and are reinforced for increasing or reducing their SCPs above or below a prestimulus level depending on discriminative stimuli. ${ }^{8}$

A recent study provided evidence for a beneficial effect of SCP training on seizure frequency showing that a number of drugrefractory patients who successfully learned to enhance and suppress cortical negativity at will within the self-regulation paradigm, also experienced a considerable reduction in seizure frequency after training. ${ }^{67}$ Despite the promising success rate associated with SCP training, there is considerable between-patient variability in the acquisition of SCP control, and a number of patients who learn to change SCPs at will do not benefit from this ability regarding changes in seizure frequency. ${ }^{67}$ Given these marked individual differences and the fact that a considerable amount of time and effort is invested in SCP training by both the patient and the therapist, it would be highly desirable to have some indication of predictors of outcome before training. Patient variables, such as medication, type of epilepsy, adjustment to illness or mood variables such as depression and anxiety are of limited usefulness in predicting SCP training outcome. ${ }^{7}$

Neuropsychological tests are valuable for the prediction of cognitive changes after surgery for epilepsy, ${ }^{9}$ and problem solving abilities were also found to be related to outcome of sensorimotor EEG normalisation biofeedback in patients with epilepsy. ${ }^{10}$ As for the cognitive processes which underlie acquisition of a SCP self-control task, it has been argued that the ability to focus attention during the brief episodes of feedback presentation (usually 8s) may play a role in the mediation of SCP selfcontrol. ${ }^{11}$ Attentional abilities might also be an important factor in SCP acquisition in patients with epilepsy, but there is still no empirical evidence for this hypothesis. This study aimed to investigate whether neuropsychological tests could help to identify (in advance of training) patients likely to acquire SCP control and to experience seizure reduction.

\section{Methods}

\section{Subjects}

Fourteen drug-refractory patients with epilepsy $(4 \mathrm{~m}, 10 \mathrm{f})$ took part in this study. Mean age was 29.9 years (range 15-47). All subjects had well-documented seizure disorders, with side and site of the focus determined by a series of clinical EEGs, CT scans and clinical evaluation. Seizure characteristics included primary generalised seizures $(n=4)$ and elementary partial $(n=1)$ or complex partial seizures $(n=8)$. One patient had secondary 

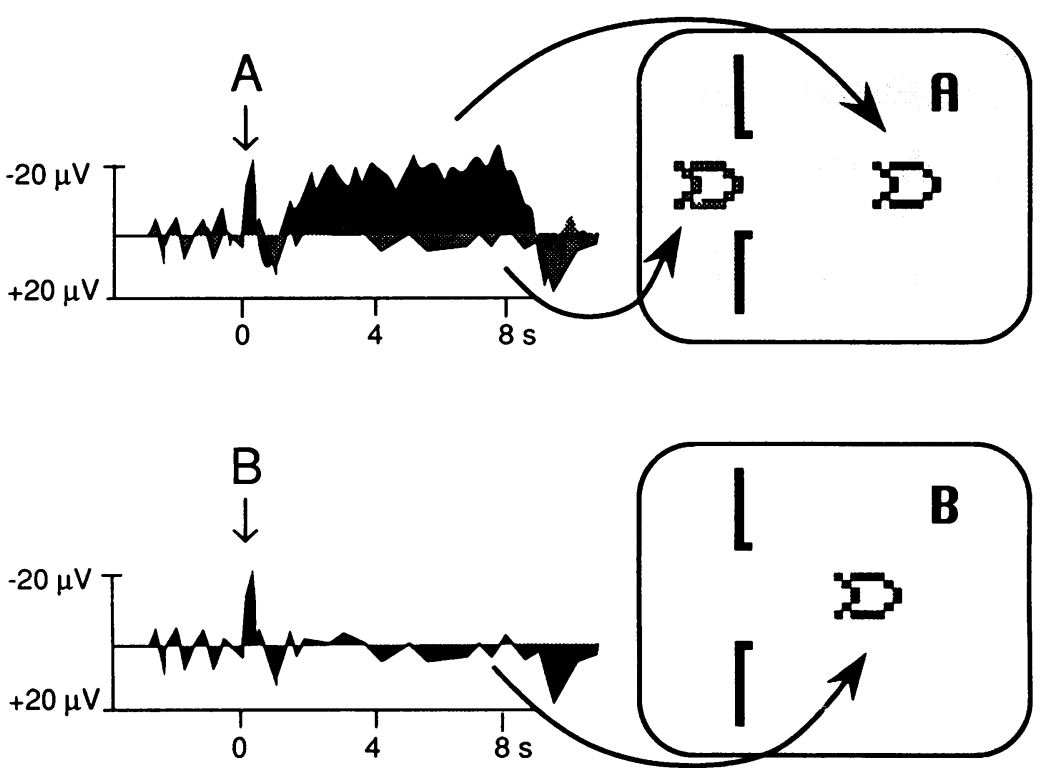

Figure Changes in slow cortical potentials during the biofeedback task. Rocket movements to the right in the presence of stimulus $A$ indicate enhancement of negativity (area shaded black, correct response in the presence of stimulus $A$ ) compared with baseline; backward movements to the left of the goal indicate negativity suppression (area shaded grey, incorrect response in the presence of stimulus $A$ ). In the presence of stimulus $B$, negativity suppression is required and rocket movements to the right indicate successful negativity suppression. and delayed reproduction of the Rey Figure ${ }^{13}$ served as measures of visuospatial memory. Attertion was assessed by means of digit spans and block- tapping spans. ${ }^{13}$ A modified version of the Wisconsin Card Sorting Test (WCST) ${ }^{14}$ was included as a measure of frontal lobe function.

\section{Biofeedback paradigm}

The paradigm used in our laboratory is described in detail elsewhere. ${ }^{68}$ In each training session, subjects received continuous visual feedback of SCPs during intervals of 8s each. EEGs were recorded from $\mathrm{Cz}$ with linked earlobes as references and a time constant of 30s. On-line artefact control procedures prevented movements of the feedback signal from being affected by eye movements or muscular artefacts. ${ }^{8}$ Feedback consisted of the movements of the outline of a "rocket-ship" appearing on television screen in front of the subject (figure). Subjects were rewarded for increasing or decreasing scalp-recorded cortical negativity as referred to pre-trial baseline values, depending upon discriminative stimuli (the letters " $A$ " and " $B$ " appearing on the screen). Forward movements of the rocket-ship indicated the required SCP-shift (for example, an increase in negativity above the mean of the pretrial baseline in the presence of the letter " $A$ "), whereas backward movements signalled poor performance (for example, reduced instead of increased negativity in the presence of the letter “ $A$ ").

Visual feedback was a linear function of the integrated EEG referred to the mean of a $4 \mathrm{~s}$ prestimulus baseline. Each feedback trial lasted 8s, with an intertrial interval of 15- 25s. Each subject completed 28 biofeedback sessions, with each session comprising 60 feedback trials in 2 blocks of 30 trials each. In addition, each subject completed 40 transfer trials ( 2 blocks of 20 trials each) in each session, during which the discriminative stimulus (" $A$ " or " $B$ ") appeared on the screen without the rocket ship giving feedback about performance. Transfer trials were included to assess learning and generalisation effects. The two trial types (negativity enhancement and suppression) varied pseudorandomly within each block.

After SCP training in the laboratory, patients were instructed to practise their strategies for achieving SCP control on " $A$ " and " $B$ " trials five times per day at home. Throughout the entire treatment period (from eight week baseline to one year follow up) patients kept diaries indicating seizure frequency and characteristics. $^{7}$ logical test battery before biofeedback training. ${ }^{9}$ The battery included verbal IQ (VIQ) and performance IQ (PIQ) derived from the subtests Vocabulary, Comprehension and Similarities (VIQ) and Block Design and Object Assembly (PIQ) of the Wechsler Adult Intelligence Scale-Revised. ${ }^{12}$ Measures of verbal memory included immediate, delayed and percentage recall (delayed/immediate recall) of two stories and verbal paired associate learning derived from the Wechsler Memory Scale. ${ }^{13}$ Immediate reproduction of the Benton figures and delayed reproduction of the Rey Figure ${ }^{13}$

\section{Analyses}

The area below the EEG curve for the $8 \mathrm{~s}$ feedback interval served as the SCP measure on each trial (figure). For each patient and session, SCPs were averaged for the two different trial types (negativity increase and suppression), for both feedback and transfer conditions separately. For each patient, the SCP difference between the two trial types was averaged across 28 sessions and evaluated by 
means of $t$ tests, and the $t$ values served as measures of overall differentiation. The linear trend of differential responding across the 28 sessions (derived from polynomial regression) served as a measure of the learning rate across training. The scores for the two blocks of feedback and transfer trials were averaged, resulting in four measures per subject: measures of overall differentiation and learning rate for feedback and transfer conditions separately.

In accordance with the procedure employed by Lantz and Sterman, ${ }^{10}$ the patient sample was split at the median separately for both performance measures and feedback and transfer trials, and the groups above and below the median were compared for neuropsychological performance. In a further analysis, test performance was compared between subjects who showed a considerable seizure reduction (to less than $67 \%$ of baseline values, $n=7$ ) and subjects with smaller or no seizure reduction (n $=6$ ). The cut-off score of $67 \%$ was based on patients' self-reports (diaries, interviews), indicating that a seizure reduction to less than two thirds of baseline level was perceived as a change meaningful to everyday life. In the group with considerable seizure reduction 4 patients were seizure free, in 2 patients seizure frequency had decreased to $33 \%$ of baseline levels, and in 1 patient to $60 \%$. The other group comprised patients with no change in seizure frequency after training $(n=3)$, and patients with a small reduction (to $86 \%(n=2)$ and to $67 \%(n=1)$ of baseline levels $)$.

\section{Results}

Neuropsychological test performance and patient variables

There were no significant correlations between performance on neuropsychological tests and medication (number of therapeutic units) or number of seizures during baseline. A significant negative correlation between verbal memory (\% recall of stories) and age ( $r=$ $-0.601, \mathrm{df}=12, \mathrm{p}<0.05)$ indicated that older patients showed poorer long-term verbal memory.

Cognitive tests and SCP acquisition during feedback trials

The median $t$ value for SCP differentiation was 1.55; the $t$ values for the subgroup above the median ranged from 1.6 to $6 \cdot 6$ (median: $2 \cdot 6$ ), the values for the subgroup below the median ranged from 1.5 to -0.1 (median: 0.6 ). The median for the linear regression coefficients was 0.35 (the coefficients above the median ranged from 0.4 to 1.9 , subgroup median: 1.0 , range below the median: 0.35 to -0.15 , subgroup median: 0 ).

As there is no German standardisation for some of the tests used in the battery described here, no definite conclusions can be reached on the level of impairments of patients with epilepsy. When published normative data derived from English- speaking samples ${ }^{12} 13$ are considered as a comparison basis, most scores were within the low normal range, with a small number of results pointing towards a mild impairment (Benton errors, Rey Recall scores).

Only one variable was related to SCP differentiation: patients with differentiation scores above the median performed better in the block-tapping span test (backward reproduction) $(t=2,38, \mathrm{p}=0.035)$. Patients with larger improvements across training did significantly better in a verbal learning test (paired associates, $t=2.54, \mathrm{p}=0.026$ ) and had longer digit spans (backward reproduction) ( $t$ $=2 \cdot 76, \mathrm{p}=0.017)$. The difference for the groups above and below the median approached significance for verbal IQ $(t=1.91, \mathrm{p}=$ $0.080)$ and a verbal memory measure (\% recall of stories, $t=2 \cdot 15, \mathrm{p}=0.052$ ).

Cognitive tests and SCP acquisition during transfer trials

The differentiation $t$ values of the subgroup above the median ranged from 1.35 to 4.5 (median: $2 \cdot 2$ ); the scores of the subgroup below the median ranged from 0.75 to -2.0 (median: $-0 \cdot 6$ ). For linear trend coefficients, the coefficients for the subgroup above the median ranged from 0.35 to 1.4 (median: 0.9 ); the scores for the subgroup below the median ranged from 0.2 to -0.6 (median: 0 ).

As for feedback trials, there was only one variable which discriminated between the two groups: patients with larger differentiation had longer block-tapping spans (forward reproduction) $(t=3.69, \mathrm{p}=0.003)$. Patients with larger improvements across sessions had longer digit spans (backward reproduction, $t=4 \cdot 06, \mathrm{p}=$ 0.002 ) and made fewer random errors during the Wisconsin Card Sorting Test $(t=2 \cdot 24, \mathrm{p}=$ $0.045)$. Other cognitive tests did not differentiate between the two groups.

\section{Cognitive tests and seizure reduction}

Only one test differentiated between the groups with successful and less successful seizure reduction: successful patients had significantly longer block-tapping spans (forward reproduction) $(t=3 \cdot 29, \mathrm{p}=0 \cdot 007)$.

\section{Discussion}

One aim of this study was to determine whether neuropsychological tests before SCP training discriminated between patients with epilepsy who were subsequently above or below the median in measures indicating acquisition of SCP control. The measures most closely and consistently related to performance during SCP control appeared to be digit spans or block-tapping spans, both measures of attention. This is not surprising as the SCP tests repeatedly required close attention and concentration for an 8 s period, during which strategies producing negativity enhancement and suppression had to be exercised and applied. ${ }^{11}$ Interestingly, virtually all traditional verbal memory and learning measures appeared to play some role in the learning rate during feedback trials. This pattern may represent attempts to recall previously successful verbalised strategies.

It should be emphasised that two issues limit 
the interpretation of our results. Firstly, in each analysis only one of the four attention span tests (digit and block-tapping spans, forward and backward reproduction) differentiated between successful and less successful subjects, and the "useful" test differed between the analyses. Secondly, a large number of $t$ tests was carried out, and a number of significant differences may have been obtained by chance. However, measures of attention consistently provided significant results and may therefore prove to be relevant.

With the exception of the block-tapping span test (forward reproduction), cognitive tests were found to be unrelated to changes in seizure frequency. These negative findings are of particular importance for exclusion criteria. Neither measures of visuospatial functioning nor frontal tests were significantly related to SCP acquisition in any analysis. This implies that patients with poor scores on these measures might still profit from this kind of therapeutic approach.

A number of procedural differences restrict direct comparisons between the present findings and those reported by the only other study in the field. ${ }^{10}$ Both studies, however, reported some relationship between general cognitive abilities and learning-related measures or seizure reduction, whilst both failed to support the idea of predictive value of visuospatial or frontal lobe tests. The lack of problem- solving task is a significant omission in the present test battery, and further studies will have to include such measures.

The overall pattern of findings tentatively suggests that epileptic patients with good scores on digit and block-tapping tests (and verbal memory and learning tests) may have a chance to acquire SCP control and profit from this ability regarding seizure frequency. Neuropsychological predictors of SCP training outcome were somewhat different from those for seizure reduction and this is consistent with the finding that factors other than the ability to control SCPs influence the implementation of this procedure in everyday life. ${ }^{7}$ Mood variables and measures of psychosocial adjustment might play a role and should be assessed more extensively than in an earlier study. ${ }^{7}$ Additional behavioural interventions (such as stress management) may aid to successfully implement SCP self-control and thereby leading to higher success rates in seizure reduction.

This research was supported by the Deutsche Forschungsgemeinschaft (SFB 307).

1 Mostofsky DI, Balaschak BA. Psychobiological control of seizures. Psycholog Bull 1977;84:723-50.

2 Dahl J, Melin L, Brorson LO, Schollin J. Effects of a broadspectrum behavior modification treatment program on spectrum behavior modification treatment program on
children with refractory epileptic seizures. Epilepsia 1985;26:303-9.

3 Forster FM. The classification and conditioning treatment of the reflex epilepsies. Int $\mathcal{f}$ Neurol 1972;9:73-86.

4 Friedman R, Rubin SR, Carlton RM, Fox MC. Behavioral control of intractable idopathic seizures: self- regulation of end-tidal carbon dioxide. Psychosomatic Medicine 1984;46:315-31.

5 Sterman MB. The role of sensorimotor rhythmic EEG activity in the etiology and treatment of generalized motor
seizures. In: Elbert T. Rockstroh B, Lutzenberger W, Birbaumer N, eds. Self-regulation of the brain and behavior. Berlin: Springer, 1984

6 Elbert T. Rockstroh B, Canavan A, Birbaumer N, Lutzenberger W, von Bulow I, Linden A. Self-regulation of slow corger W, von Bulow I, Linden A. Self-regulation of slow cortical potentials and its role in epileptogenesis. In: Carlson J, Seifert R, eds. Biobehavioral

7 Rockstroh B, Elbert T, Birbaumer N, Wolf P, DüchtingRöth A, Daum I, Dichgans J, Lutzenberger W. Cortical self-regulation in patients with epilepsies. Epilepsy Research 1992 (in press).

8 Elbert T, Rockstroh B, Lutzenberger W, Birbaumer N. Biofeedback of slow cortical potentials. Electroenceph Clin Neurophysiol 1980;48:293-301.

9 Powell GE, Polkey CE, McMillan T. The new Maudsley series of temporal lobectomy. Short-term cognitive effects. Br f Clin Psychol 1985;24:109-24.

10 Lantz DL, Sterman MB. Neuropsychological assessment of subjects with uncontrolled epilepsy: effects of EEG fubjects with uncontrolled epilepsy: effects

11 Roberts LE, Birbaumer N, Rockstroh B, Lutzenberger W, Elbert T. Self-report during feedback regulation of slow
cortical potentials. Psychophysiology 1989;26:392-403.

cortical potentials. Psychophysiology 1989;26:392-403.
12 Wechsler D. Wechsler Adult Intelligence Scale (revised). New York: Psychological Corporation, 1981.

13 Powell GE. The relationship between intelligence and verbal and spatial memory. F Clin Psychol 1979;35:336-40.

14 Nelson HE. A modified card sorting test sensitive to frontal lobe defects. Cortex 1976;12:313-24. 